\title{
Confocal chromatic sensor for displacement monitoring in research reactor
}

\author{
Marion Agoyan ${ }^{1,2}$, Gary Fourneau ${ }^{1}$, Guy Cheymol ${ }^{1}$, Ayoub Ladaci ${ }^{1}$, Hicham Maskrot ${ }^{1}$, Christophe \\ Destouches $^{3}$, Damien Fourmentel ${ }^{3}$, Sébastien Gérand ${ }^{4}$, Jérôme Gaillard-Groléas ${ }^{4}$, Matthieu \\ Desjacques $^{4}$, Sylvain Girard ${ }^{2}$ and Aziz Boukenter ${ }^{2}$. \\ ${ }^{1}$ CEA, DES/ISAS/DPC/SEARS/LISL, Saclay, France \\ ${ }^{2}$ Université Jean Monnet, CNRS, Graduate School Optics Institute, Laboratoire Hubert Curien UMR \\ 5516, Saint-Etienne, France \\ ${ }^{3} \mathrm{CEA}, \mathrm{DES} / \mathrm{IRESNE} / \mathrm{DER} / \mathrm{SPESI}$, Cadarache, France \\ ${ }^{4}$ STIL Marposs, Aix-en-Provence, France \\ Corresponding author: marion.agoyan@,cea.fr
}

\begin{abstract}
Confocal chromatic microscopy is an optical technique allowing measuring displacement, thickness, and roughness with a sub-micrometric precision. Its operation principle is based on a wavelength encoding of the object position. Historically, the company STIL based in the south of France has first developed this class of sensors in the 90's. Of course, this sensor can only operate in a sufficiently transparent medium in the used spectral domain. It presents the advantage of being contactless, which is a crucial advantage for some applications such as the fuel rod displacement measurement in a nuclear research reactor core and in particular for cladding-swelling measurements. The extreme environmental conditions encountered in such experiments i.e. high temperature, high pressure, high radiations flux, strong vibrations, surrounding turbulent flow can affect the performances of this optical system. We then need to implement mitigation techniques to optimize the sensor performance in this specific environment. Another constraint concerns the small volume available in the irradiation rig next to the rod to monitor, implying the challenge to conceive a miniaturized sensor able to operate under these constraints.
\end{abstract}

Keywords - Confocal Chromatic Sensor, Pressurized Water Reactor, Radiation-Induced-Attenuation, Radiation-InducedRefractive-Index-Change, Temperature, Optical design.

\section{INTRODUCTION}

$\mathrm{T}$ HIS work is made in the framework of the instrumentation for nuclear applications (INSNU) project, aiming to develop instrumentation and sensors for experiments in the Jules Horowitz reactor (Cadarache, France). Among other technologies are developed optical sensors: fiber Bragg gratings [1], fiber-optic distributed sensors to measure temperature and strain, pyrometric measurement [2], Fabry-Perot extensometer [3] and confocal chromatic sensor: a displacement sensor which is the object of this article.

After a certain operating time, fuel stresses the rod and leads to its deformation [4]. We develop the confocal chromatic sensor in order to monitor this deformation. This technology allows avoiding contact between the probe and the rod when performing the measurement and thus we will avoid risking scratching and initiating rod rupture.

As the sensor will be in an irradiation rig simulating the operation in a Pressurized Water Reactor (PWR) we have to consider its typical environment. This means temperature about $350^{\circ} \mathrm{C}$, pressure of 150 bar. Very high radiation levels are expected: fluence of $10^{19} \mathrm{n}, \mathrm{fast} / \mathrm{cm}^{2}$ and gamma dose about GGy. We will also have to consider turbulent flow $(3 \mathrm{~m} / \mathrm{s})$ of corrosive water. Indeed, at such temperatures, water becomes corrosive for an optical window. The confocal chromatic sensor is set in front of a rod in the nuclear reactor. Then, three sensors located around the rod may be necessary to monitor its displacement due to possible buckling of the fuel rod. For this specific measurement of fuel rod swelling in a research reactor, all the interrogation part of the instrumentation will be deported by optical fiber outside the reactor core.

The overall principle of a confocal chromatic sensor is based on the physical concept of chromatism, with the change in optical refractive index with wavelength. Usually, chromatism is an optical aberration to avoid, but not in our case. When focusing a white light source with an optical system, not all the wavelengths are focusing at the same spot on the light propagation axis. In our system, a white source is focused through an optical chromatic system on the fuel rod. The rod's surface, made of an oxidized alloy of zirconium, is reflective enough to be considered as a diffusing surface. Therefore, the light hitting the rods goes back through the whole system. It is only partially reinjected in the fiber because of the spatial filtering due to the small fiber core diameter. This filtering depends on how the beam is focused on the surface in the optically conjugate plane. That produces a spectral selection, which can be visualized on an optical spectrum analyzer at the transport fiber output. Thus, we will detect a peak centered on a particular wavelength. If the fuel rod was swelling, we would then detect a change of the peak central wavelength. As we have an encoding between wavelengths and distance, we can then 
deduce of how many micrometers the rod swelled. The difference between the initial wavelength and the final one would give an equivalent value of distance's difference (Fig. 1).

Here are the targeted specifications for this sensor:

- Diameter of the sensor: $4 \mathrm{~mm}$ (mechanic), $3 \mathrm{~mm}$ (optics)

- Total length $<10 \mathrm{~cm}$

- Accuracy : 1-10 $\mu \mathrm{m}$

- Working distance (distance between the sensor output and the rod): $0.5 \mathrm{~mm}$

- $\quad$ Measuring range (chromatism): $1.5 \mathrm{~mm}$

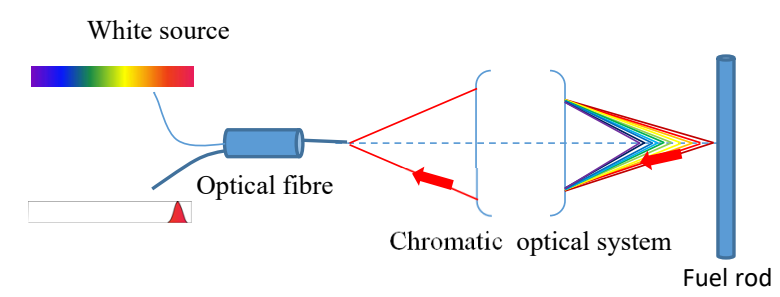

a)

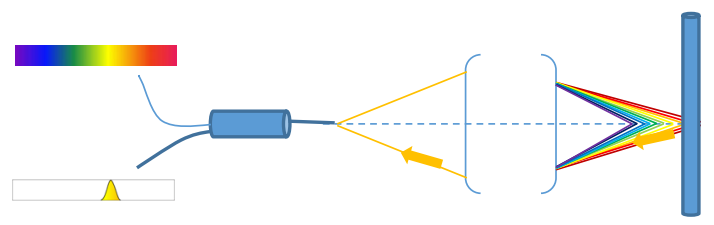

b)

Figure 1: confocal chromatic sensor global principle. a) confocal chromatic in front of a fuel rod before swelling, b) after swelling

Some of the main challenges of this confocal chromatic sensor design are listed hereafter:

- The size of the sensor (both length and diameter) has to be very small to fit the reduced available space and to prevent perturbations on the cooling flow. However, as a wide measuring range (chromatism) is expected, this is more difficult to obtain with such small dimensions. The optical design software -Zemax- is exploited to conceive and optimize the sensor optical system.

- When a glass bulk is heated, its length is modified (thermal dilatation), as well as its refractive index, which will lead to displacement of the focusing points and therefore to measurement errors. Data relative to refractive index dependence to temperature from the literature are needed and if none is available, dedicated experiments will be mandatory.

- Radiation darkens the optical glasses [3] and optical fibers [5]. Moreover, large fast neutron fluence is also known to modify the glass refractive index, at least of the silica [6] [7]. Depending on the amplitudes of these effects, additional measurement errors can occur up to a loss of the signal. We then have to find radiation tolerant optical glasses and acquire data about their radiation responses.

In this paper we will present a brief state-of-the-art regarding the effects of radiation and thus explain the need to collect data during an irradiation campaign in BR2 (Belgium Reactor2) in 2022 (irradiation "TESCA"). We first present interferometric measurement technique to measure the changes of refractive index of a glass under irradiation. First, the setup is validated in laboratory conditions (variations with temperature only) before its future implementation in the 2022 irradiation campaign. In a second part, we focus on the optical design of the confocal chromatic sensor, describing the main challenges and the solutions to overcome them.

\section{Change OF REFRACTIVE INDEX AND ATTENUATION WITH RADIATIONS}

\section{A. State of the art}

For many years [8], we have known that radiations influence optical fiber and optical glass properties. For our targeted environments, the two most impacting radiation effects on optical fiber and glasses are the Radiation Induced Attenuation (RIA) and the Radiation Induced Refractive Index Change (RIRIC).

RIA corresponds to the glass darkening under radiations related to an increased attenuation of the signal. RIA is caused by point defects created in the optical fiber core and cladding [5]. This is quite a complex phenomenon, depending on both the structural and optical properties of the point defects and on how the harsh environment constraints (dose, fluence, dose rate, and temperature) influence their generation mechanisms. Moreover, the RIA can recover after radiations through the thermal or photo bleaching of the point defects. For our confocal chromatic design, we need to choose glasses or optical fibers presenting lower RIA in the spectral domain of interest. For optical fibers, few data are available in the literature at high neutron fluence with a lack of information on the behavior at high temperature and/or a large spectral domain [9-16]. We will select a pure silica (or slightly F-doped silica) core optical fiber with a metallic coating to withstand the high temperature. About bulk glasses for the chromatic optical system, some cerium doped radiation-hardened glasses are considered, but no data at the very high targeted dose levels is available. We also plan to use pure-silica glass, which the behavior in a high radiation environment is better known. Finally, we plan to operate at wavelengths between 800 and $1200 \mathrm{~nm}$ where the optical fibers and glasses present the lowest RIA levels. This is also compatible with operation in water that is strongly absorbing above $1200 \mathrm{~nm}$.

RIRIC is mostly observed under neutron exposure and corresponds to the radiation induced change of the refractive index. This is also a topic with little data available in the literature. Moreover, temperature will also induce a variation of refractive index [17].

For the development of the confocal chromatic sensor, we need to consider the glass darkening, if we want to perform the measurement with a better signal to noise ratio. We also have 
to consider the change of refractive index when glasses are exposed to high fast neutron fluence and/or high temperature in order to avoid measurement errors. Most of all, we want to reduce the degradation of the focusing quality of the chromatic optical system.

In order to consider glass darkening and refractive index change we have designed an experiment to collect data on candidate optical glasses. That will be one of the objectives of the TESCA experiment in Belgium Reactor 2 in 2022.

\section{B. Experimental setups and preliminary results at high temperature}

We developed two small measurement systems aiming to collect online data when performing TESCA irradiation. First one is designed to collect RIA data and the second one for refractive index change measurements, more precisely for optical path measurement $n(\lambda$, radiations,$T) \times$ $L($ radiations,$T)$.

We have developed an opto-mechanical device bringing a light beam at the exact center of a mirror curvature. Light is fully reinjected in the fiber except when the RIA of a glass sample (placed in between) attenuates the beam (Fig. 2). We use an halogen lamp to illuminate the system and we collect the output signal on an Optical Spectrum Analyzer (OSA). RIA at a particular wavelength corresponds to the ratio of intensity going in the system and the one going out.

Before irradiation, we had to check if the setup is functioning well under high temperature i.e. that heating the device will not unsettle the optical alignment. The results of a preliminary test are presented in Fig. 3. Blue curve represents the time evolution of temperature (we aim $350-400{ }^{\circ} \mathrm{C}$ ) and the orange one illustrates the power evolution at $970 \mathrm{~nm}$. This illustrates the good stability of the setup through time and so with temperature. We conclude that the setup is suitable for radiation experiments at high temperature.

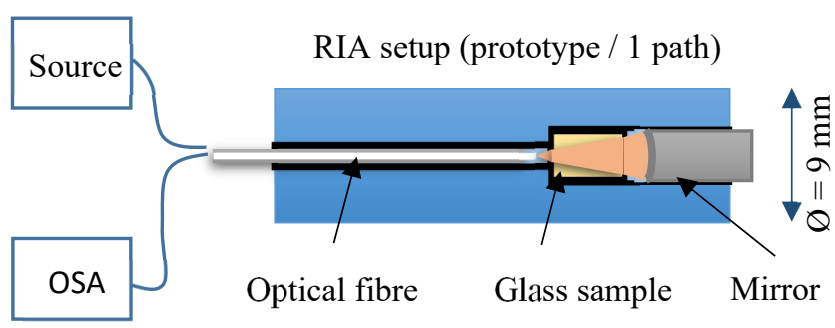

Figure 2: RIA setup

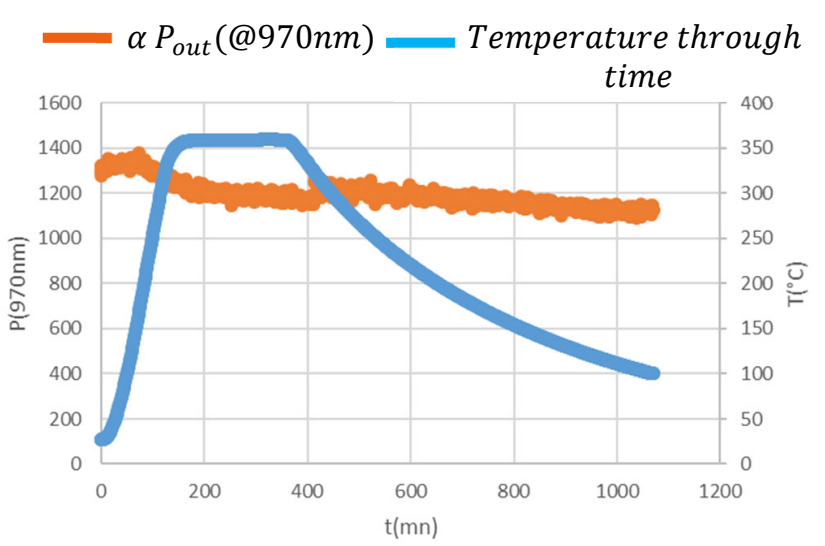

Figure 3: Stability of RIA setup with temperature

We also developed a setup in order to measure the variations of the refractive index with radiations. The first objective is indeed to measure the refractive index change. But since high neutron fluence is likely to produce density change leading to dimension change, the targeted online measurement of refractive index $(n)$ therefore becomes an optical length $(n \times L)$ measurement. We are also preparing pre and post-irradiation measurement of glass samples to decorrelate variations of $\mathrm{n}$ and L. The setup is represented in the Fig.4. This is an interferometric measurement; we get a channeled spectrum from the interferences coming from both sides of the glass sample. From that spectrum and after signal processing, we get the value of the glass optical length which allows deducing the refractive index if the length is known. We have not yet tested the setup under radiative environment but the setup performances were estimated on different glasses, at different wavelengths and at different temperatures up to $350^{\circ} \mathrm{C}$.

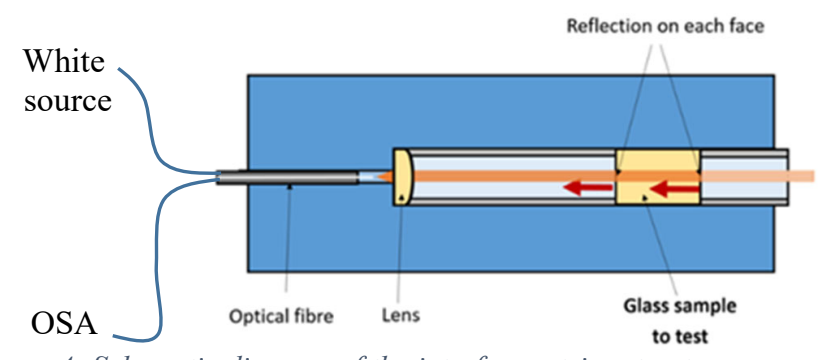

Figure 4: Schematic diagram of the interferometric setup to measure optical path or refractive index change under radiative environment.

Table 1 compares the theoretical values of refractive index and the data collected from our experimental setup.

\begin{tabular}{|c|c|c|c|c|c|}
\hline$\lambda[\mathrm{nm}]$ & $\begin{array}{c}\text { CO 79- } \\
40\end{array}$ & SF6G05 & LF5G19 & BK7G18 & K5G20 \\
\hline $1010_{\text {th }}$ & 1.4503 & 1.7789 & 1.5813 & 1.5102 & 1.5132 \\
\hline $1010_{\text {exp }}$ & 1.4524 & 1.7798 & 1.5769 & 1.5116 & 1.5176 \\
\hline $1110_{t h}$ & 1.4491 & 1.7761 & 1.5796 & 1.5089 & 1.5119 \\
\hline $1110_{\text {exp }}$ & 1.4512 & 1.7768 & 1.5752 & 1.5104 & 1.5164 \\
\hline $1210_{t h}$ & 1.4479 & 1.7737 & 1.5782 & 1.5076 & 1.5108 \\
\hline $1210_{\text {exp }}$ & 1.4500 & 1.7745 & 1.5738 & 1.5090 & 1.5151 \\
\hline
\end{tabular}

Table 1: Refractive index at room temperature 
We can observe a difference of about $10^{-3}$ for absolute values of refractive index at 1010,1110 and $1210 \mathrm{~nm}$ for optical glasses CO 79-40, SF6G05, LF5G19, BK7G18 and K5G20. The theoretical refractive index values of our glasses at room temperature (not under radiation environment) are obtained from the glassmaker Corning and Schott datasheets. We might observe some differences due to different glass batches. Other possible sources for these differences might be a shift in the wavelength calibration of the OSA or an error when measuring the length of the sample. All these errors will remain constant during the irradiation campaign and should not affect the ability of the developed system to measure the refractive index changes.

This can be confirmed by measuring the evolution of refractive index for a particular glass, at a specific wavelength, with the temperature aiming $350^{\circ} \mathrm{C}$. By doing so, we also tested the robustness of the device with temperature. When increasing it, we have been able to observe relative changes in the optical path in the range of few $10^{-3}$. We have obtained data that is not yet available in the literature, and which is necessary to design the targeted optical system operating at high temperature. The setup is then almost ready to perform online measurement under a high radiative environment in 2022 .

\section{OptiCAL DESIGN}

We discuss in this part, the optical design of the optical sensor for the fuel rod swelling monitoring in a research core reactor. At this design stage, the application requires a small size of the sensor. Temperature and radiation constraints are considered.

We require reaching $1.5 \mathrm{~mm}$ measuring range while keeping a good focus quality. Indeed, when the light reflects on the rod and goes back on the optical fiber, we need to read a wavelength with the best selectivity possible in order to give a swelling measurement with the $\sim 1-10 \mu \mathrm{m}$ accuracy. We then have to optimize our optical design with a compromise between chromatism and spot quality while fulfilling the specifications in terms of diameter and total track.

If we look at a common lens commercialized by Thorlabs, LB4879, f= 35.0 mm, Ф1" UV Fused Silica Bi-Convex Lens, for instance- with an object numerical aperture of 0.12 , we get the results described below.

The initial configuration for this bi-convex lens is near to a $2 \mathrm{f}-2 \mathrm{f}$ and we get:

- An image numerical aperture of 0.10 i-e. a paraxial magnification of about.1.15

- $\quad$ A focal shift (chromatism) of $1878 \mu \mathrm{m}$

- A very bad quality of spot due to a high spherical aberration. In order to improve this point we could consider the lens curvatures as variables and even aspherize one face of the lens.

Now, if we consider the same lens but with smaller diameter, the numerical aperture is reduced leading to reduced wavelength selectivity too. To avoid that we can change the configuration (object-image position). By doing so, we will not get better results in terms of chromatism and selectivity.
Table 2 illustrates the impossibility to design a confocal chromatic sensor with a single lens of the required size. This table shows the results obtained from the Thorlabs -LB4879 lens with an object numerical aperture of 0.12 and for wavelengths between 800 and $1200 \mathrm{~nm}$. The Design referenced as " 1 " on this table refers the $2 \mathrm{f}-2 \mathrm{f}$ configuration spoken below and design " 2 " were obtained after optimization aiming a bigger image numerical aperture, a good spot quality with aspherization on the first surface of the lens and both curvatures set as variables -also aiming smaller diameters of the lens. On table 2, D1 refers to the distance between the source and the first surface of the lens and D2 corresponds to the distance between the second surface of the biconvex lens and the Image at $800 \mathrm{~nm}$.

\begin{tabular}{|c|c|c|}
\hline Design & 1 & 2 \\
\hline D1 [mm] & 63.749 & 10,41 \\
\hline D2 @ 800 nm [mm] & 70.486 & 71,1185 \\
\hline Thickness [mm] & 7.440 & 7.440 \\
\hline Curvature 1 [mm] & 30.96 & 2,5 \\
\hline Curvature 2 [mm] & -30.96 & $-2,5$ \\
\hline Conic of Curvature 1 & $\mathrm{x}$ & -0.882 \\
\hline $\begin{array}{c}\text { Clear Semi Diameter 1 } \\
{[\mathbf{m m}]}\end{array}$ & $\mathbf{1 3}$ & $\mathbf{1 , 3 1 9}$ \\
\hline $\begin{array}{c}\text { Clear Semi diameter 2 } \\
{[\mathbf{m m}]}\end{array}$ & $\mathbf{1 3}$ & $\mathbf{0 . 3 3 8}$ \\
\hline & $\mathrm{x}$ & $\mathrm{V}$ \\
\hline Spot diagram & 5 & 2,755 \\
\hline Airy Radius [ $\mu \mathrm{m}]$ & $\mathbf{1 8 7 8}$ & $\mathbf{7 0}$ \\
\hline $\begin{array}{c}\text { Chromatism (Focal shift) } \\
{[\boldsymbol{\mu m}]}\end{array}$ & 0,10 & 0,23 \\
\hline Numerical Aperture (Image) & & \\
\hline
\end{tabular}

Table 2: Thorlabs-LB4879, $f=35 \mathrm{~mm}, \Phi 1^{\prime \prime}$ UV Fused Silica Bi-

Convex Lens. Design 1: starting design. Design 2: constraint on diameter of optics with aspherization.

As we can see, with a single lens (even with a more chromatic glass), it is impossible to design a small diameter confocal chromatic sensor. We have to work on a more complex design, which finally will have to combine different radiation hardened glasses, especially the most dispersive ones.

\section{CONCLUSIONS}

We are developing a confocal chromatic sensor in order to measure fuel rod's swelling in a research nuclear reactor. We have discussed the main scientific and technological challenges that have to be overcome: temperature, radiations and optical design under strict specifications. Temperature changes the refractive index of about $10^{-3}$. Radiation also induces a change in refractive index and a glass darkening. Small dimensions of the sensor probe are limiting the measuring range resulting from chromatism.

New data on the behavior of bulk glasses under radiation are expected to result from the TESCA irradiation (2022, in the $\mathrm{BR} 2$ research reactor). We have developed miniature devices in order to measure RIA and the variations of the optical path of some glass samples under harsh environment. These devices will be set in the research reactor's core for online measurements; they have been tested and validated under high temperature. The optical path-measuring device gives data on 
the refractive index of glasses at high temperature. These data are necessary for the design of the confocal chromatic sensor, and are not yet all available in literature. Our next step will be the irradiation campaign in 2022 at BR2. While we will consolidate refractive index change setup and signal processing in order to get a better accuracy, confocal chromatic sensor design on Zemax with silica and a mix of silica and radiationhardened (and more dispersive) glasses will be achieved.

\section{REFERENCES}

[1] S. Nehr et al., "On-line monitoring of multiplexed femtosecond Fiber Bragg Gratings exposed to high temperature and high neutron fluence", 26th International Conference on Optical Fiber Sensors, OFS-26, Poster presentation, Lausanne, Switzerland, 24-28 September, 2018

[2] B. Bouvry et al., "Multispectral pyrometry for surface temperature measurement of oxidized Zircaloy claddings," Infrared Physics and Technology, vol. 83, pp. 78-87, 2017

[3] G. Cheymol, A. Verneuil, P. Grange, H. Maskrot and C. Destouches, "HighTemperature Measurements With a Fabry-Perot Extensometer," in IEEE Transactions on Nuclear Science, vol. 67, no. 4, pp. 552-558, April 2020, doi: 10.1109/TNS.2020.2975707.

[4] P. Van Uffelen, G. Pastore, "Oxide Fuel Performance Modeling and Simulation”, Comprehensive, Nuclear Materials, vo. 2, 2020, pp 363-416.

[5] S. Girard et al., "Overview of radiation induced point defects in silicabased optical fibers," Reviews in Physics, 2019, doi: https://doi.org/10.1016/j.revip.2019.100032

[6] Primak, "Fast-neutron-induced changes in quartz and vitreous silica", Phys.Rev.B 110 (6),1240-1254(1958).

[7] L. Remy, G. Cheymol, A. Gusarov, A. Morana, E. Marin and S. Girard, "Compaction in Optical Fibres and Fibre Bragg Gratings Under Nuclear Reactor High Neutron and Gamma Fluence," in IEEE Transactions on Nuclear Science, vol. 63, no. 4, pp. 2317-2322, Aug. 2016, doi: 10.1109/TNS.2016.2570948.

[8] David L. Griscom, " $\gamma$ and fission-reactor radiation effects on the visiblerange transparency of aluminium-jacked, all-silica optical fibers," Journal of Applied Physics, vol. 80, no. 4, May 1996, pp 2142-2155

[9] T. Allanche, "Effect of high radiation doses (MGy) on light Emitting Diodes and optical glasses", HAL id: tel-03215958, May 2021

[10] T. Shikama, T. Kakuta, M. Narui, T. Sagawa, H. Kayano, "Optical properties in fibers during irradiation in a fission reactor", J. Nucl. Mater 225 (1995) 324-327

[11] D. W. Cooke, B. L. Benett, E. H. Farnum, "Optical absorption of neutron irradiated silica fibers", J. Nucl. Mater, May 1996, pp 214-218

[12] T. Kakuta, T. Shikama, M. Narui, T. Sagawa "Behavior of optical fibers under heavy irriadiations”, Fus. Eng. Des. 51-52 (2000) 179-183

[13] B. Brichard, P. Borgemans, A. F. Fernandez, K. Lammens, M. Decréton, "Radiation effect in silica optical fiber exposed to intense mixed neutrongamma radiation field", IEEE Trans. Nucl. Sci, vol. 48, no. 6, Nov. 2001, pp2069-2073

[14] T. Kakuta, T. Shikama, T. Nishitani, B.Brichard, A. Krassilinikov, A. Tomashuk, S. Yamamoto, S.Kasai "Round-robin irradiation test of radiation resistant optical fibers for ITER diagnostic application", Journal of nuclear materials 307-311 (2002) 1277-1281
[15] G. Cheymol, H. Long, J. F. Villard, B. Brichard, "High level gamma and neutron irradiation of silica optical fibers in CEA OSIRIS nuclear reactor", IEEE. Trans. Nucl. Sci, vol. 55, no. 4, Aug. 2008, pp 2252-2258

[16] P. F. Kashaykin, A. L. Tomashuk, S.A. Vasiliev, A.D. Ignatyev, A.A. Shaimerdenov, Yu. V. Ponkratov, T. V. Kulsartov, Y. A. Kenzhin, Sh. Kh. Gizatulin, T. K. Zholdybayev, Y. V. Chikhray, S. L. Semjonov, "Radiation resistance of single-mode optical fibres with view to in-reactor applications", Nuclear Materials and Energy 27 (2021) 10098.

[17] C. Z. Tan, "Temperature dependence of refractive index of glassy $\mathrm{SiO} 2$ in the infrared wavelength range", Journal of Physics and Chemistry of Solids 61 (2000) 1315-1320. 